\title{
Population-based Cervical Cancer Screening Using High- risk HPV DNA Test and Liquid-based Cytology in Northern Thailand
}

\author{
Sumalee Siriaunkgul' ${ }^{1}$, Jongkolnee Settakorn'1, Kornkanok Sukpan ${ }^{1}$, Jatupol \\ Srisomboon ${ }^{2}$, Prapaporn Suprasert ${ }^{2}$, Nongyao Kasatpibal ${ }^{3}$, Surapan \\ Khunamornpong ${ }^{1 *}$
}

\begin{abstract}
Background: Northern Thailand is a region with a high cervical cancer incidence. Combined high-risk HPV (hrHPV) DNA testing and cytology (co-testing) has increasingly gained acceptance for cervical cancer screening. However, to our knowledge, data from a population-based screening using co-testing have not been available in this region. This study therefore aimed to evaluate the performance of cytology and hrHPV test in women in northern Thailand. Materials and Methods: Cervical samples were collected for hybrid capture 2 (HC2) testing and liquid-based cytology from women aged 30 to 60 years who were residents in 3 prefectures of Chiang Mai in northern Thailand between May and September 2011. Women with positive cytology were referred to colposcopy, while women with positive for $\mathrm{HC} 2$ only were followed for 2 years. Results: Of 2,752 women included in this study, 3.0\% were positive in both tests, $4.1 \%$ for $\mathrm{HC} 2$ only, and $1.3 \%$ had positive cytology only. At baseline screening, positive $\mathrm{HC} 2$ was observed in $70.6 \%$ among cytology-positive women compared with $4.3 \%$ among cytology-negative women. The prevalence of positive $\mathrm{HC} 2$ or cytology peaked in the age group 35-39 years and was lowest in the age group 55-60 years. High-grade squamous intraepithelial lesion or worse lesions (HSIL+) were histologically detected in $23.5 \%$ of women with positive baseline cytology and in $9.8 \%$ of women with positive baseline HC2 only on follow-up. All women with histologic HSIL+ had positive baseline HC2. Conclusions: The hrHPV test is superior to cytology in the early detection of high-grade cervical epithelial lesions. In this study, the prevalence of histologic HSIL+ on follow-up of women with positive hrHPV test was rather high, and these women should be kept under careful surveillance. In northern Thailand, hrHPV testing has a potential to be used as a primary screening test for cervical cancer with cytology applied as a triage test.
\end{abstract}

Keywords: Cervical cancer screening - liquid-based cytology - HPV DNA test - Thailand

Asian Pac J Cancer Prev, 15 (16), 6837-6842

\section{Introduction}

Cervical cancer is an important health problem, being one of the most common cancers in women worldwide and in Thailand. Chiang Mai, located in northern Thailand, is one of the regions with the highest cervical cancer incidence in Southeast Asia (age standardized incidence rate of 28.9 per 100,000 women) (Curado et al., 2007). Effective Cervical cancer screening could reduce cervical cancer incidence and increase life expectancy of women by detection of early-stage cancer or precancerous (intraepithelial) lesions, which could be effectively treated (Ito et al., 2013; Jin et al., 2013). Human papillomavirus (HPV) infection, particularly of high-risk genotypes, is recognized to be a necessary cause of almost all cervical cancers (Walboomers et al., 1999).

Cervical cytology has long been a mainstay in cervical cancer screening. Liquid-based cytology (LBC) has been developed as an alternative method instead of conventional cervical cytology (Pap test) and has been demonstrated to offer benefits over Pap test, particularly in the reduction of unsatisfactory samples and in further testing for HPV (Ronco et al., 2006; Ogilvie et al., 2012; Pan et al., 2013). Testing for HPV DNA has recently gained increasing acceptance in cervical cancer screening. Hybrid capture 2 (HC2) is the most widely used commercial DNA test for high-risk HPV (hrHPV) and has been clinically validated (Arbyn et al., 2012). Although hrHPV DNA testing is found to be more sensitive than cervical cytology in the detection of cervical epithelial lesion, cytology still has a higher specificity (Cuzick et al., 2006; Jin et al., 2013; Pan et al., 2013). A combination of cytology and hrHPV test (co-testing) has been established as a highly sensitive method of cervical cancer screening in women aged 30

${ }^{1}$ Department of Pathology, ${ }^{2}$ Department of Obstetrics and Gynecology, Faculty of Medicine, ${ }^{3}$ Faculty of Nursing, Chiang Mai University, Chiang Mai, Thailand*For correspondence: skhunamo@yahoo.com 
years or older (Castle et al., 2012). Women with negative co-test have a very low risk of cervical cancer and can be rescreened after an interval of 3-to 5-years (Castle et al., 2012; Massad et al., 2013).

Due to the variation in the prevalence of HPV infection and cervical lesions between areas to areas, the screening strategy may be variable in different regions. Studies on cervical cancer screening using HPV test showed remarkable variation in positive prevalence, which was reported to be as high as $40 \%$ in some study (Carestiato et al., 2006). However, the information that could be reliably used as a basis for strategic decision and resource allocation in cancer screening and prevention should derive from a population-based study in each region (Sharma et al., 2013). In Thailand, the information on cervical cancer screening using hrHPV test is very limited, and, to our knowledge, such data from a population-based study has not been available.

The aim of this study was to evaluate the performance of LBC and hrHPV test in a population-based cervical cancer screening in women in northern Thailand. We also evaluated the correlation between the results of hrHPV test, cytology, and final histology in this women population.

\section{Materials and Methods}

This study was approved by the institutional Ethics Committee of the Faculty of Medicine Chiang Mai University (study code: PAT-11-02-07A-14-X). The study population comprised women who were residents in 3 prefectures (Sankumpang, Mae-on, and Sarapee) of Chiang Mai. These women attended a cervical cancer screening scheduled by the Ministry of Health between May and September 2011. Women were eligible to participate in this study if they were aged between 30 and 60 years and had any previous sexual relationship. Excluded were women who were pregnant or menstruating, had received hysterectomy, had a previous history of cervical precancerous lesion or cancer, or had a previous Pap test within the last 3 months. Informed consent was obtained from all participants.

Cervical specimen was collected for cytology and hrHPV test by trained nurses, using a plastic spatula and a cytobrush. The cervical samples were transferred from collecting devices into PreservCyt solution (Cytyc Corporation, Boxborough, MA, USA) for ThinPrep LBC preparation (Hologic, Marlborough, MA, USA) and for HC2 test (Qiagen, Hilden, Germany). According to the manufacturer's information, HC2 is designed to detect 13 high-risk HPV genotypes $(16,18,31,33,35,39,45$, $51,52,56,58,59$ and 68). Positive HC2 test is defined with a quantitative threshold of relative light unit/positive control (RLU/PC) ratio $\geq 1.0$.

Cervical cytology slides were separately examined by 2 cytopathologists (J.S. and K.S.) blinded to the HC2 results. The final cytology results, based on a consensus of both pathologists, were reported according to the 2001 Bethesda System for reporting cervical cytology. The cytological results were classified for analysis as; a) negative, b) atypical squamous cells of undetermined significance (ASC-US)/low-grade squamous intraepithelial lesion (LSIL), and c) atypical squamous cells, cannot exclude HSIL, or worse (ASC-H + ). Women who had positive cytology (ASC-US or worse) were referred to colposcopy. Women who tested positive with $\mathrm{HC} 2$ but had negative cytology were scheduled for 2-year follow-up protocol which included LBC after 6 and 18 months, and co-testing after 12 and 24 months (Figure $1)$. Women who had positive cytology in any follow-up testing or had persistent positive HC2 after a 2-year period were referred to colposcopy. A final histologic diagnosis in this study referred to the worst histologic lesion detected in any tissue specimen of each patient, obtained either by biopsy, conization, or hysterectomy. The final histologic diagnoses were classified for analysis as; a) negative (no epithelial lesion identified), b) LSIL (or

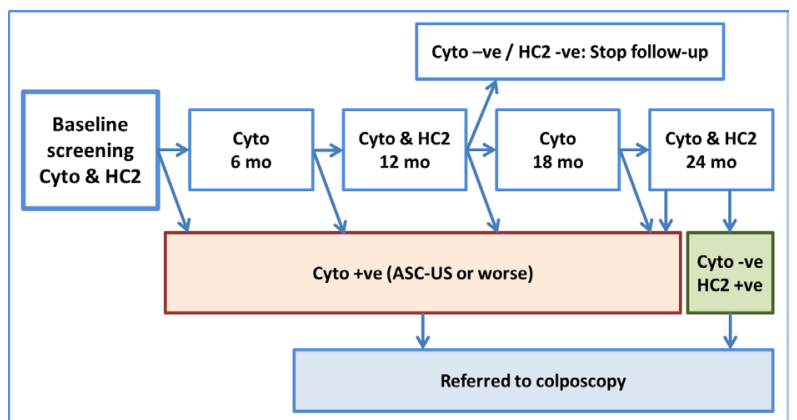

Figure 1. Flow Chart of The Protocol for Follow-up and Management after the Baseline Screening

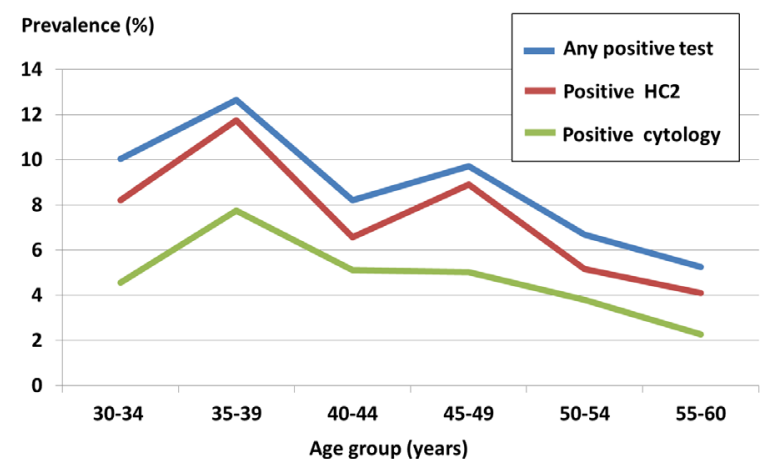

Figure 2. Prevalence of Positive Tests Within Each Age Group at the Baseline Screening

Table 1. Age Distribution of 2,752 Women Stratified by Baseline Results of Cytology and HC2

\begin{tabular}{|c|c|c|c|c|c|c|}
\hline \multirow[t]{2}{*}{ Age (years) } & \multirow[t]{2}{*}{$\begin{array}{l}\text { Total No. } \\
\qquad(\%)\end{array}$} & \multirow[t]{2}{*}{$\begin{array}{l}\text { Positive either } \\
\text { test }(\%)\end{array}$} & \multicolumn{2}{|c|}{$\begin{array}{l}\text { Positive cytology } \\
(\mathrm{n}=119)\end{array}$} & \multicolumn{2}{|c|}{$\begin{array}{c}\text { Negative cytology } \\
(n=2,633)\end{array}$} \\
\hline & & & Positive $\mathrm{HC} 2(\%)$ & Negative HC2 (\%) & Positive HC2 (\%) & Negative HC2 (\%) \\
\hline $30-39$ & $551(20.0)$ & $64(27.7)$ & $21(25.0)$ & $7(20.0)$ & $36(32.1)$ & 487 (19.3) \\
\hline $40-49$ & $1,105(40.2)$ & $100(43.3)$ & $43(51.2)$ & $13(37.1)$ & $44(39.3)$ & $1,005(39.8)$ \\
\hline $50-59$ & $1,096(39.8)$ & $67(29.0)$ & $20(23.8)$ & $15(42.9)$ & $32(28.6)$ & $1,029(40.9)$ \\
\hline Total & $2,752(100)$ & $231(100)$ & $84(100)$ & $35(100)$ & $112(100)$ & $2,521(100)$ \\
\hline
\end{tabular}


cervical intraepithelial neoplasia [CIN] 1), and c) highgrade squamous intraepithelial lesion or worse (HSIL+ or CIN2+).

The data were analyzed by STATA version 11 (StataCorp LP, College Station, TX, USA). Association between variables was evaluated by Chi square test or Fisher Exact test as appropriate. Comparison between groups was assessed by risk ratio with $95 \%$ confidence interval. A p value $<0.05$ was considered statistically significant.

\section{Results}

There were 2,761 women aged 30-60 years who received cervical screening tests. Nine women $(0.3 \%)$ were excluded due to unsatisfactory cytology specimen (all were HC2-negative), resulting in a total of 2,752 women enrolled into this study (mean age 46.6 \pm SD 7.5 years). Of these 2,752 women, 231 women $(8.4 \%)$ had either positive $\mathrm{HC} 2$ or positive cytology (ASC-US+) at the baseline screening round (Table 1). In this group with positive test results, 84 women $(3.0 \%)$ had positive both HC2 and cytology, whereas 112 women (4.1\%) had positive $\mathrm{HC} 2$ only and 35 women $(1.3 \%)$ had positive cytology only (total positive HC2 rate $7.1 \%$, and total positive cytology rate $4.3 \%$ ).

The prevalence of positive test results within each age group (5-year interval) is shown in Figure 2. The highest prevalence of positive tests was observed in the age group 35-39 years $(12.7 \%$ for any test, $11.8 \%$ for $\mathrm{HC} 2$, and $7.8 \%$ for cytology). After age 50 years, the prevalence of positive tests declined gradually and the age group 55-60 years had the lowest prevalence $(5.3 \%$ for any test, $4.1 \%$ for $\mathrm{HC} 2$, and $2.3 \%$ for cytology).

Table 2 shows a correlation between cytology results and $\mathrm{HC} 2$ results at the baseline screening round. Positive cytology was observed in $42.9 \%$ among HC2-positive women (84 of 196) compared with $1.4 \%$ among HC2negative women $(35$ of 2556) $(\mathrm{p}<0.001)$. Positive HC2 was observed in $70.6 \%$ among cytology-positive women (84 of 119) compared with $4.3 \%$ among cytology-negative women $(112$ of 2633) $(\mathrm{p}<0.001)$. The HC2-positive risk ratio of each positive cytology category (ASC-US, LSIL, and ASC-H+) was significantly higher than that of negative cytology group ( $\mathrm{p}<0.001$ each).

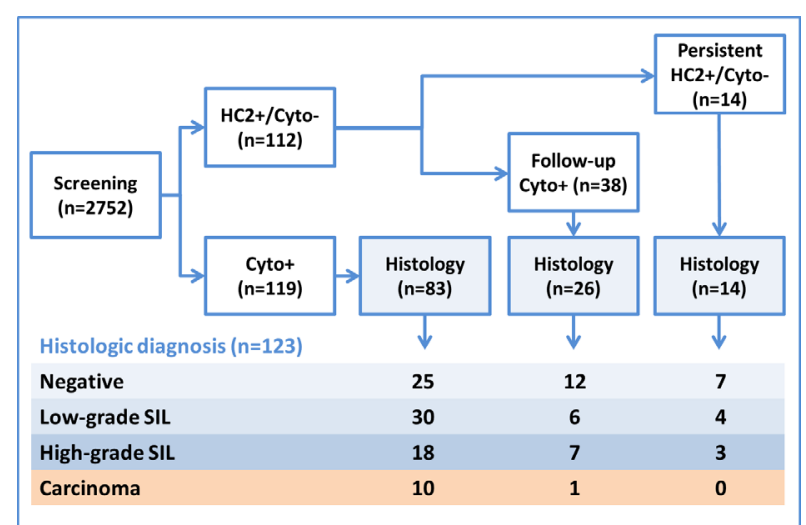

Figure 3. Flow Chart and Final Histologic Diagnoses of Cervical Tissue Obtained from 123 Women
Within a 2-year period of follow-up, a total of 171 women had either positive cytology (157 women: 119 from baseline round and 38 from follow-up) or persistent positive HC2 only (14 women). Cervical tissue specimens were obtained in a total of 123 women (Figure 3 ). The final histological diagnoses in these 123 women included negative result in 44 women (35.8\%), LSIL in 40 women $(32.5 \%)$, and HSIL+ in 39 women $(31.7 \%)$. Among 39 women with histologic HSIL+, 28 women had HSIL, whereas the remaining 11 women had early-stage cervical cancer including squamous cell carcinoma (8 women) and adenocarcinoma (3 women). The cytology results that led to colposcopy in these 11 women with cancer included ASC-US in 3 women, ASC-H in 3 women, HSIL in 4 women, and squamous cell carcinoma in 1 woman. Based on the baseline screening round, 28 of 2752 women (1.0\%) were found to have histologic HSIL+. All these 28 women also had positive HC2 test. Among 112 women with positive HC2 but negative cytology at baseline, 11 women (9.8\%) were found on follow-up to have histologic HSIL+ (including adenocarcinoma in 1 woman). The prevalence of histologic HSIL+ in these women was significantly higher than that of overall population at baseline screening (9.8\% vs $1.0 \%, \mathrm{p}<0.001)$.

A correlation between cytology result, baseline $\mathrm{HC} 2$, and final histologic diagnosis among 109 women with available histologic specimens is shown in Table 3 . Of 86 women with ASC-US/LSIL cytology, histologic HSIL+ was detected in 15 women $(17.4 \%)$. Of 23 women with ASC-H+ cytology, 21 women $(91.3 \%)$ had histologic HSIL+. All women with histologic HSIL+ had positive $\mathrm{HC} 2$ at baseline screening. Among women who had ASC-US/LSIL cytology, those with positive HC2 had a significantly higher rate of histologic HSIL+ than those with negative $\mathrm{HC} 2(23.1 \%$ vs $0 \%, \mathrm{p}=0.017)$.

We also evaluated whether the performance of $\mathrm{HC} 2$ in our screening population could be improved by a change in the cut-off values of RLU/PC ratio or not. The detection rate of histologic HSIL+ by different cut-off RLU/PC ratios is shown in Table 4. While increasing the cut-off

Table 2. Correlation between Baseline Cytology and HC2 Results in 2,752 Women

\begin{tabular}{|c|c|c|c|}
\hline Cytology & $\begin{array}{c}\text { Positive } \\
\text { HC2 }\end{array}$ & $\begin{array}{c}\text { Negative } \\
\text { HC2 }\end{array}$ & $\begin{array}{l}\text { Risk ratio } \\
(95 \% \mathrm{CI})\end{array}$ \\
\hline Negative $(\%), \mathrm{n}=2,633$ & $(4.3)$ & $2521(95.7)$ & Reference \\
\hline Positive $(\%), \mathrm{n}=119$ & $84 \quad(70.6)$ & $35(29.4)$ & $16.6(13.4-20.6)$ \\
\hline ASC-US (\%), $\mathrm{n}=63$ & $30 \quad(47.6)$ & $33(52.4)$ & $11.2(8.1-15.4)$ \\
\hline $\operatorname{LSIL}(\%), \mathrm{n}=30$ & $28 \quad(93.3)$ & $2(6.7)$ & $21.9(17.9-26.9)$ \\
\hline ASC-H or worse $(\%), n=26$ & $626(100)$ & $0 \quad(0)$ & $23.5(19.6-28.2)$ \\
\hline
\end{tabular}

Table 3. Correlation between Cytology Result, Baseline HC2, and Final Histology in 109 Women

\begin{tabular}{lcccc}
\hline Histology & Total No. & \multicolumn{3}{c}{ Cytology } \\
& & $\begin{array}{c}\text { ASC-US/LSIL with } \\
\text { negative HC2 (\%) }\end{array}$ & $\begin{array}{c}\text { ASC-US/LSIL with } \\
\text { positive HC2 (\%) }\end{array}$ & $\begin{array}{c}\text { ASC-H or } \\
\text { worse (\%)* }\end{array}$ \\
\hline Negative & 37 & $12(57.1)$ & $25(38.5)$ & 0 \\
LSIL & 36 & $9(42.9)$ & $25(38.5)$ & $2(4.5)$ \\
HSIL or worse & 36 & 0 & $15(23.1)$ & $21(95.5)$ \\
Total & 109 & $21(100)$ & $65(100)$ & $23(100)$ \\
\hline *All women with ASC-H or worse cytology were HC2-positive
\end{tabular}

Asian Pacific Journal of Cancer Prevention, Vol 15, 2014 
Sumalee Siriaunkgul et al

Table 4. The Performance of Baseline HC2 Using Different Cut-Off Values in The Detection of Histologic HSIL or Worse Lesions in 123 Women

\begin{tabular}{llcc}
\hline HC2 result by RLU/PC ratio & \multicolumn{2}{c}{ Histologic HSIL or worse } \\
& & Present & Absent \\
\hline Cut-off ratio $\geq 1.0$ & Positive $(\%)$ & $39(38.2)$ & $63(61.8)$ \\
& Negative (\%) & 0 & $21(100)$ \\
Cut-off ratio $\geq 2.0$ & Positive (\%) & $37(39.4)$ & $57(60.6)$ \\
& Negative (\%) & $2(6.9)$ & $27(93.1)$ \\
Cut-off ratio $\geq 3.0$ & Positive (\%) & $35(39.7)$ & $53(60.2)$ \\
& Negative (\%) & $4(11.4)$ & $31(88.6)$ \\
\hline
\end{tabular}

ratio to 2.0 or 3.0 resulted in only a slightly increase of the detection rates of histologic HSIL+ (from $38.2 \%$ to $39.4-39.7 \%$ ), this resulted in missing $6.9 \%$ and $11.4 \%$ of the patients with histologic HSIL+ with the cut-off values of 2.0 and 3.0 , respectively.

\section{Discussion}

The significance of the present study is that our data were obtained from a population-based cervical cancer screening and would be more reliable than that from institution-or hospital-based studies, which may be affected by certain selection bias. This study also includes a correlation with the histologic diagnoses and follow-up results, which reflects the performance of screening tests in the population. To our knowledge, there was no previous study on population-based screening using combined hrHPV test and cytology in Thailand. A previous population-based HPV study in Thai women focused on HPV detection and genotyping (39 highand low-risk genotypes) in cervical cell samples using polymerase chain reaction (PCR) techniques (Sukvirach et al., 2003), which might not represent the performance of hrHPV test in cancer screening. That study included 1,741women from 2 sites: Lampang in northern Thailand and Songkhla in southern Thailand. Overall prevalence of HPV infection was $6.3 \%$, but the prevalence was higher in Lampang $(8.0 \%)$ than in Songkhla (3.8\%) (Sukvirach et al., 2003). Histologic correlation or follow-up results were not available in that study.

The prevalence of positive hrHPV testing appears to be variable depending on geographic regions and the types of HPV test (Franceschi et al., 2006; Leinonen et al., 2008; Arbyn et al., 2009; Sharma et al., 2013). Considering population-based studies in which $\mathrm{HC} 2$ testing was used, the HC2-positive prevalence of $7.1 \%$ in our study is comparable to the reported prevalence from countries in Europe and North America (6.3-8.1\%) (Cuzick et al., 2003; Ronco et al., 2006; Leinonen et al., 2008; Castle et al., 2009; Ogilvie et al., 2012; Zorzi et al., 2013), while it is higher than the prevalence in the Netherlands $(4.5 \%)$ (Bulkmans et al., 2007) but is lower than that in India (10.3-16.2\%) (Sankaranarayanan et al., 2009; Pandey et al., 2012; Rai et al., 2014) and China (9.9-27.5\%) (Zhao et al., 2010; Li et al., 2013). Population-based studies for HPV detection by PCR techniques also have demonstrated high overall HPV prevalence in Latin America and Africa, where cervical cancer incidence is high (Herrero et al.,
2000; Franceschi et al., 2006).

In an institution-based study from central Thailand with 14,747 participants using similar screening tests to our study (LBC and HC2) (Swangvaree et al., 2010), the prevalence of positive $\mathrm{HC} 2$ and positive cytology was rather similar to that in our study population $(8.2 \%$ vs $7.1 \%$ and $3.7 \%$ vs $4.3 \%$, respectively). However, the previous study (Swangvaree et al., 2010) reported a lower HC2-positive rate in women with positive cytology (60.7\%) compared with our study (70.6\%). In addition, the HC2-positive rate among women with negative LBC in that study $(6.3 \%)$ was higher than the rate in our study $(4.3 \%)$. The lack of a correlation with histology and follow-up results is a limitation in the evaluation of the performance of the screening tests in that previous study (Swangvaree et al., 2010). The HC2-positive rate in LBC-negative women in the current study is within the reported range of $3.4-5.4 \%$ in other population-based studies in developed countries, which suggests that the performance of LBC in our study is comparable to that of the other studies (Ronco et al., 2006; Bulkmans et al., 2007; Castle et al., 2009; Ogilvie et al., 2012; Katki et al., 2013). The HC2-positive rate in LBC-negative women was reported as high as $11.2 \%$ in China (Zhao et al., 2010). In developing countries, HPV prevalence in women with negative cytology may also be correlated with cervical cancer incidence (Sharma et al., 2013).

In the present study, the prevalence of positive hrHPV test or cytology peaked in the age group 35-39 years, which is rather different from several other studies in which hrHPV prevalence gradually decline after the age of 25 to 30 years (Cuzick et al., 2003; Leinonen et al., 2008; Arbyn et al., 2009; Swangvaree et al., 2010; Zorzi et al., 2013). However, studies in China have shown a "two peak" pattern of hrHPV prevalence, with the first peak in the age group 15-24 years (prevalence 16.2$18.7 \%$ ) and the second peak in the age group 35-39 years (prevalence 16.0-18.6\%) (Li et al., 2013). As our study did not include women younger than 30 years, whether the other peak prevalence in the younger age group also exists in our population could not be confirmed. Although the explanation for variation in the age group with peak hrHPV prevalence is uncertain, it might be partly contributed by the difference in sexual behavior among different populations. Women aged 50-60 years represent the group with the lowest positive rate of either cytology or HC2 in our study. The lower hrHPV prevalence in women aged 55 to 60 years observed in our study is similar to that observed in most other regions except for some countries in Latin America where hrHPV prevalence shows a second increase after the age of 55 years (Franceschi et al., 2006; Ferreccio et al., 2013).

In this study, $9.8 \%$ of women with positive HC2 only at the baseline screening had histologic HSIL+ on a 2-year follow-up period. This prevalence was higher than the range of 3-7\% reported in previous studies from the other regions (Ferreccio et al., 2013; Jin et al., 2013). Women with positive hrHPV test but negative cytology have an increasing risk of cervical precancerous lesions and cancer over time. In a large US study, the 5-year risk of histologic HSIL+ in these women was $10.0 \%$, 
while the 5 -year risk of cervical cancer was $0.34 \%$ with a high proportion of adenocarcinoma (53\% of cancer cases) (Katki et al., 2013). In the most recent guidelines for management of women with positive HPV test but negative cytology (Massad et al., 2013), repeat co-testing at a 1-year interval is acceptable. Women with any positive test result in repeat co-testing should be referred to colposcopy, whereas those with negative tests can be rescreened after a 3-year interval. Alternatively, triage of these women with genotyping test for HPV16/HPV18 is also acceptable (Massad et al., 2013).

The rate of positive HC2 within each cytologic category correlated with the severity of results, ranging from $4.3 \%$ in negative cytology to $100 \%$ in ASC-H+ group (Table 2). Among women with ASC-US/LSIL cytology, those with positive $\mathrm{HC} 2$ had a significantly higher rate of histologic HSIL+ compared with those with negative HC2 (Table 3). The correlation between cytology and HC2 results and histologic diagnosis supports that HPV test could be useful to stratify the risk for histologic HSIL+ in women who have ASC-US/LSIL cytology in northern Thailand. Alternatively, based on the higher sensitivity of $\mathrm{HC} 2$ than cytology, it might be considered that $\mathrm{HC} 2$ could be used as a primary screening test in women in northern Thailand, and cytology could be used to triage HC2-positive women and to stratify the risk for histologic HSIL+ (Cuzick et al., 2006; Castle et al., 2012; Jin et al., 2013). While HPV testing is more objective compared with cytology testing, effective cervical cytology screening requires a well-organized program with high standards of quality control (Dudding and Crossley, 2013), which might not be possible in all regions particularly the areas with limited-resource settings (Rashid et al., 2013). With HPV testing as a primary screening test, the screening performance could be more uniformly reproduced across different regions. However, the rather high cost of HPV testing is an important limitation for this approach in developing countries (Othman and Othman, 2014). Technical advances might help reduce the cost of HPV testing and lead to acceptable cost-effectiveness of the test. In addition to a well-organized screening program, an effective recall system for women with positive test for further investigations also contributes to successful cervical cancer prevention in developing countries (Rashid et al., 2013; Wang et al., 2014).

The cut-off RLU/PC ratio for a positive $\mathrm{HC} 2$ result has been a controversial aspect. Women with a high RLU/PC ratio are more likely to have cervical lesions (Junyangdikul et al., 2013). Previous large studies suggest that a cut-off ratio of 2.0 may be more appropriate for cervical cancer screening in the populations with low cervical cancer incidence (Ronco et al., 2006; Zorzi et al., 2013). Based on a rather limited number of patients in our study, increasing the cut-off value would lead to missing a substantial number of women with histologic HSIL+. Considering that northern Thailand is a region with a high incidence of cervical cancer and that a rather high rate of histologic HSIL+ including cancers among women with ASC-US and LSIL Pap test results (14\% and 32\%, respectively) has been highlighted in the previous studies from this region (Kantathavorn et al., 2008; Kiatiyosnusorn et al., 2010), the usual cut-off ratio of 1.0 seems optimal for the use in cervical cancer screening in northern Thailand.

In conclusion, hrHPV test is superior to cytology in the early detection of high-grade cervical epithelial lesions. The prevalence of positive hrHPV test in women in northern Thailand was within intermediate range compared with the other reports. However, there was a rather high prevalence of histologic HSIL+ on followup of women who had positive hrHPV test but negative cytology. This finding indicates that women in this region with positive HPV test should be under careful surveillance. In northern Thailand, hrHPV testing has a potential to be used as a primary screening test for cervical cancer and cytology could be used as a triage test.

\section{Acknowledgements}

This study was supported by The National Research University Project under Thailand's Office of the Higher Education Commission, Chiang Mai University, and Faculty of Medicine, Chiang Mai University. We thank Suthida Intaraphet, MNS, for assistance with statistical analysis.

\section{References}

Arbyn M, Benoy I, Simoens C, et al (2009). Prevaccination distribution of human papillomavirus types in women attending at cervical cancer screening in Belgium. Cancer Epidemiol Biomarkers Prev, 18, 321-30.

Arbyn M, Ronco G, Anttila A, et al (2012). Evidence regarding human papillomavirus testing in secondary prevention of cervical cancer. Vaccine, 30, 88-99.

Bulkmans NW, Berkhof J, Rozendaal L, et al (2007). Human papillomavirus DNA testing for the detection of cervical intraepithelial neoplasia grade 3 and cancer: 5-year followup of a randomised controlled implementation trial. Lancet, 370, 1764-72.

Carestiato FN, Silva KC, Dimetz T, Oliveira LH, Cavalcanti SM (2006). Prevalence of human papillomavirus infection in the genital tract determined by hybrid capture assay. Braz J Infect Dis, 10, 331-6.

Castle PE, de Sanjose S, Qiao YL, et al (2012). Introduction of human papillomavirus DNA screening in the world: 15 years of experience. Vaccine, 30, 117-22.

Castle PE, Fetterman B, Poitras N, et al (2009). Five-year experience of human papillomavirus DNA and Papanicolaou test cotesting. Obstet Gynecol, 113, 595-600.

Curado MP, Edwards B, Shin HR, et al (2007). Cancer incidence in five continents, Vol. IX. Lyon: IARC Scientific Publications No.160.

Cuzick J, Clavel C, Petry KU, et al (2006). Overview of the european and north american studies on HPV testing in primary cervical cancer screening. Int J Cancer, 119, 1095-101.

Cuzick J, Szarewski A, Cubie H, et al (2003). Management of women who test positive for high-risk types of human papillomavirus: the HART study. Lancet, 362, 1871-6.

Dudding N, Crossley J (2013). Sensitivity and specificity of HPV testing: what are the facts? Cytopathology, 24, 283-8.

Ferreccio C, Barriga MI, Lagos M, et al (2013). Screening trial of human papillomavirus for early detection of cervical cancer in Santiago, Chile. Int J Cancer, 132, 916-23.

Franceschi S, Herrero R, Clifford GM, et al (2006). Variations in the age-specific curves of human papillomavirus prevalence 
in women worldwide. Int J Cancer, 119, 2677-84.

Herrero R, Hildesheim A, Bratti C, et al (2000). Populationbased study of human papillomavirus infection and cervical neoplasia in rural Costa Rica. J Natl Cancer Inst, 92, 464-74.

Ito K, Tsunematsu M, Satoh K, Kakehashi M, Nagata Y (2013). Effectiveness of cervical cancer screening based on a mathematical screening model using data from the Hiroshima Prefecture Cancer Registry. Asian Pac J Cancer Prev, 14, 4897-902.

Jin XW, Lipold L, McKenzie M, Sikon A (2013). Cervical cancer screening: what's new and what's coming? Cleve Clin J Med, 80, 153-60.

Junyangdikul P, Tanchotsrinon W, Chansaenroj J, et al (2013). Clinical prediction based on HPV DNA testing by hybrid capture 2 (HC2) in combination with liquid-based cytology (LBC). Asian Pac J Cancer Prev, 14, 903-7.

Kantathavorn N, Kietpeerakool C, Suprasert P, et al (2008). Clinical relevance of atypical squamous cells of undetermined significance by the 2001 bethesda system: experience from a cervical cancer high incidence region. Asian Pac J Cancer Prev, 9, 785-8.

Katki HA, Schiffman M, Castle PE, et al (2013). Five-year risks of CIN 3+ and cervical cancer among women who test Pap-negative but are HPV-positive. J Low Genit Tract Dis, 17, 56-63.

Kiatiyosnusorn R, Suprasert P, Srisomboon J, et al (2010). Highgrade histologic lesions in women with low-grade squamous intraepithelial lesion cytology from a region of Thailand with a high incidence of cervical cancer. Int J Gynaecol Obstet, 110, 133-6.

Leinonen M, Kotaniemi-Talonen L, Anttila A, et al (2008). Prevalence of oncogenic human papillomavirus infection in an organised screening population in Finland. Int J Cancer, 123, 1344-9.

Li J, Huang R, Schmidt JE, Qiao YL (2013). Epidemiological features of human papillomavirus (HPV) infection among women living in mainland China. Asian Pac J Cancer Prev, 14, 4015-23.

Massad LS, Einstein MH, Huh WK, et al (2013). 2012 updated consensus guidelines for the management of abnormal cervical cancer screening tests and cancer precursors. J Low Genit Tract Dis, 17, 1-27.

Ogilvie GS, Krajden M, van Niekerk DJ, et al (2012). Primary cervical cancer screening with HPV testing compared with liquid-based cytology: results of round 1 of a randomised controlled trial-the HPV FOCAL Study. Br J Cancer, 107, 1917-24.

Othman N, Othman NH (2014). Detection of human papillomavirus DNA in routine cervical scraping samples: use for a national cervical cancer screening program in a developing nation. Asian Pac J Cancer Prev, 15, 2245-9.

Pan QJ, Hu SY, Zhang X, et al (2013). Pooled analysis of the performance of liquid-based cytology in populationbased cervical cancer screening studies in china. Cancer Cytopathol, 121, 473-82.

Pandey S, Mishra M, Chandrawati (2012). Human papillomavirus screening in north Indian women. Asian Pac J Cancer Prev, 13, 2643-6.

Rai AK, Das D, Kataki AC, et al (2014). Hybrid capture 2 assay based evaluation of high-risk HPV status in healthy women of north-east India. Asian Pac J Cancer Prev, 15, 861-5.

Rashid RM, Dahlui M, Mohamed M, Gertig D (2013). Adapting the Australian system: is an organized screening program feasible in Malaysia?--an overview of cervical cancer screening in both countries. Asian Pac J Cancer Prev, 14, 2141-6.

Ronco G, Giorgi-Rossi P, Carozzi F, et al (2006). Human papillomavirus testing and liquid-based cytology in primary screening of women younger than 35 years: results at recruitment for a randomised controlled trial. Lancet Oncol, 7, 547-55.

Ronco G, Segnan N, Giorgi-Rossi P, et al (2006). Human papillomavirus testing and liquid-based cytology: results at recruitment from the new technologies for cervical cancer randomized controlled trial. J Natl Cancer Inst, 98, 765-74.

Sankaranarayanan R, Nene BM, Shastri SS, et al (2009). HPV screening for cervical cancer in rural india. $N$ Engl J Med, 360, 1385-94.

Sharma M, Bruni L, Diaz M, et al (2013). Using HPV prevalence to predict cervical cancer incidence. Int J Cancer, 132, 1895-900.

Sukvirach S, Smith JS, Tunsakul S, et al (2003). Populationbased human papillomavirus prevalence in lampang and Songkla, thailand. J Infect Dis, 187, 1246-56.

Swangvaree SS, Kongkaew P, Rugsuj P, Saruk O (2010). Prevalence of high-risk human papillomavirus infection and cytologic results in Thailand. Asian Pac J Cancer Prev, $11,1465-8$.

Walboomers JM, Jacobs MV, Manos MM, et al (1999). Human papillomavirus is a necessary cause of invasive cervical cancer worldwide. J Pathol, 189, 12-9.

Wang Y, Yu YH, Shen K, et al (2014). Cervical cancer screening and analysis of potential risk factors in 43,567 women in Zhongshan, China. Asian Pac J Cancer Prev, 15, 671-6.

Zhao FH, Lin MJ, Chen F, et al (2010). Performance of highrisk human papillomavirus DNA testing as a primary screen for cervical cancer: a pooled analysis of individual patient data from 17 population-based studies from china. Lancet Oncol, 11, 1160-71.

Zorzi M, Del Mistro A, Farruggio A, et al (2013). Use of a highrisk human papillomavirus DNA test as the primary test in a cervical cancer screening programme: a population-based cohort study. BJOG, 120, 1260-7. 